\title{
Numerical modeling of the droplet vaporization for design and operation of liquid pulsed CVD
}

\author{
$\underline{\text { Raphaël Boichot }}^{1}$, Susan Krumdieck ${ }^{2}$. \\ ${ }^{1}$ Univ. Grenoble Alpes, SIMAP, F-38000 Grenoble, France. CNRS, SIMAP, F-38000 Grenoble, France. \\ ${ }^{2}$ Department of Mechanical Engineering, Univ. of Canterbury, Christchurch, New Zealand.
}

\begin{abstract}
This article presents an approach for modeling the vaporization of droplets of solvent and precursor mixture under vacuum in the pulsed-pressure CVD process. The pulsed direct liquid injection apparatus with ultrasonic atomizer has been demonstrated as a controllable and reliable alternative to the bubbler and carrier gas system. Design variables include the reactor base and peak pressures, solution injection volume, precursor concentration, and reactor surface temperature in the flash evaporation zone. The numerical modeling solves mass, heat and momentum continuity equations on liquid droplets and is intended to evaluate the relative roles of the physical chemistry properties and reactor parameters in the fast vaporization of droplets. The sensitivity analysis proposed here shows that the vaporization time of the pulsed liquid CVD system is mainly dependent on the heating available in the flash evaporation zone, then on the thermodynamic properties of the liquid solution. The practical example of TTIP (Titanium Isopropoxide) delivery is presented to select the solvent and design the thermal conditions for optimal evaporation efficiency and sharpest reactor pressure rise.
\end{abstract}

Keywords: Pulsed pressure CVD, Titanium oxide, flash vaporization, metalorganic precursor delivery, numerical modeling.

Corresponding author: Raphaël BOICHOT, SIMAP / Phelma Bâtiment Recherche, 1130 rue de la piscine 38402 Saint Martin d'Hères.

E-mail : Raphael.boichot@simap.grenoble-inp.fr

Tel : +33476826537, Fax : +33476826677 


\section{Introduction}

Researchers have explored a large number of technology variations of chemical vapor deposition (CVD) processing over the past thirty years [1]. Most of the CVD variations involve different ways to deliver the precursor vapor to the substrate surface, referred to as the delivery system. Producing reactant vapor flows is challenging, and mass transport of the vapor to and away from the surface is the most costly problem in CVD engineering. It is particularly difficult to control and measure the flows, control the temperature and mass transport through the reactor, and most importantly, control the arrival rate at the deposition surface so that uniform flux across the substrate is achieved. A tremendous engineering effort is required, often taking more than a decade, to develop a manufacturing system for a particular CVD science regime, which includes the thin film material, its properties and function within a device [1].

The pulsed-pressure metalorganic CVD (pp-MOCVD) process was developed with the objective of controlling the injection process, and using gas expansion as the main transport phenomena in order to have better conversion efficiency and arrival flux uniformity on complex shaped substrates [2]. The main feature of the process is direct liquid injection into the reactor chamber via an ultrasonic nozzle in discreet timed shots, without a carrier gas. The reactor pressure rises rapidly from a base pump-down pressure to a maximum pressure when the precursor solution flashes, then the reactor is pumped back down to the base pressure. The spike in pressure causes the mass transport process in the reactor to be dominated by expansion rather than viscous flow. The expansion mass transport process has been studied by experimentation [3] and numerical simulation [4]. Expansion and diffusion of precursor molecules are much faster processes than the pumping rate of the vacuum system, which is characterized by the pump-down time constant (the ratio of reactor volume to vacuum volumetric pump rate). This pulsed pressure operation can produce high flux rate to complex shaped surfaces over the pulse cycle, depending on the maximum pressure achieved and the sharpness of the pressure rise [5]. In previous studies we made the assumption that the rise in pressure when injecting precursors/solvent liquid mixtures is instantaneous, and that all of the solution is vaporized, e.g. $100 \%$ vaporization efficiency. This assumption implies that the vaporization is not kinetically limited at the time scale of an injection period, which fits with the experimental measurement of the vaporization time of typically less than a few microseconds.

In this paper, we explore the physics of low-pressure vaporization by developing a thermodynamic model of the pp-MOCVD flash evaporation process. The results of the model will be used to inform selection of the solvent and design and operation of the injection system in order to achieve the most complete liquid-vapor conversion in the shortest time. Although liquid precursors are common in MOCVD, with liquid injection and aerosolassisted processes commonly used by researchers, there have been few published studies of the droplet evaporation in MOCVD [6]. The most comprehensive studies were about evaporation issues in bubbler systems, which are a much different evaporation situation $[7,8]$. Vaporization of droplets is a well-known phenomena used in combustion, spray drying, and plasma spray coating. Liquid droplets are also used to produce solid powders [9]. However, none of these phenomena are the same as the pulsed injection into a vacuum with the objective of producing a sharp spike in pressure of precursor vapor.

This contribution aims to provide a straight-forward phenomenological model of the droplet evaporation for two possible solvents with vastly different vaporization properties. Much of 
our previous research and development work on the pp-MOCVD process has used $\mathrm{TiO}_{2}$ deposition from titanium isopropoxide (TTIP) in toluene solvent. TTIP is one of the few MOCVD precursors with physical properties reported in the literature. The modeling study will compare n-hexane and toluene as solvents.

\section{Numerical modeling of the liquid pulsed-CVD process}

\subsection{Initial Conditions}

The liquid droplets in the pulsed CVD process are suddenly released into vacuum from a piezoelectric nebulizer. At the initiation of the vaporization process, the liquid droplet is at a very-far-from-equilibrium state where the reactor pressure is much lower than the vapor pressure of the solvent at its initial temperature. The initial pressure is assumed to be near zero relative to the droplet pressure. The reactor volume is subject to continuous pump-down from the vacuum system. The numerical model starts from the moment of emergence of a known mass of droplet into the vacuum, with initial liquid temperature equal to the solution supply bottle at room temperature.

\subsection{Numerical Modeling Assumptions for OD Solution}

1. The mass flux is calculated in the molecular regime over the whole vaporization process.

The Knudsen number $(\mathrm{Kn})$ represents the probability of vapor-vapor molecule collisions compared to the probability of vapor-surface collisions. $\mathrm{Kn}$ is usually referenced to the diameter of the reactor in the direction of flow. In pp-MOCVD, the droplets are the relevant collision surface, so $\mathrm{Kn}$ is referenced to the droplet diameter, e.g. $\mathrm{Kn}=$ mean free path / droplet diameter. During the entire droplet vaporization $\mathrm{Kn}>0.01$ in the case study conditions. In consequence, only the Hertz-Knudsen mass flux is considered, as no resistance to mass transfer around droplets can exist in molecular regime.

2. The droplet internal temperature is constant.

This point was verified by a finite difference coding of the internal droplet temperature during the flash evaporation step (the most intensive step in terms of thermal flux). The transient heat transfer equation was solved for hexane in spherical coordinates with a boundary heat flux imposed by the phase change and a radial heat balance imposed by the mass transport. No noticeable temperature difference between the surface and the core of droplets was observed.

3. The droplet precursor and solvent concentrations are constant (well-mixed condition into liquid phase).

Once released into vacuum, the local accumulation of solvent or precursor on the droplet evaporating surface is not taken into account, due to very large uncertainties of the internal mixing phenomena due to external drag. Droplets are considered internally well-mixed. It is clearly a debatable assumption of our modeling. 
4. The freezing of droplets is not taken into account.

As the droplets should be made of pure and particle-free liquids (no nuclei for crystallization), the existence of sub-cooled phases in the time scale of a vaporization time is plausible.

5. Physical properties of liquid phases are assumed constant except vapor pressure.

Vapor pressure of both solvents and TTIP are strong functions of temperature, but all other thermal properties of liquid phases are assumed constant. Thermal data of liquid phases and vapor pressure relationships with temperature originate from the NIST Chemistry WebBook [10] or literature [11].

6. Heat transfer and temperature assumptions.

Due to the 0D statement of the problem, droplet radiation heating is assumed to be from a uniform temperature of surrounding walls with view factor of 1. Emissivity of all solid and liquid surfaces is assumed to be unity, as is the droplet surface absorptivity. Due to its very high heat diffusivity (low gas density), vapor in the reactor is considered to be in equilibrium with the reactor wall temperature. The un-heated reactor wall is assumed to be at room temperature.

\section{Reactor design specifications for the case study}

The case study conditions for the modeling are based on a current reactor configuration with pumping rate: $15 \mathrm{~m}^{3} / \mathrm{h}$, evaporation coefficients: 0.1 , reactor volume: $10 \mathrm{~L}\left(0.01 \mathrm{~m}^{3}\right)$, droplet size: $18 \mu \mathrm{m}$, initial mass: $0.1 \mathrm{~g}$, initial mixture temperature: $300 \mathrm{~K}$ and reactor wall temperature: $300 \mathrm{~K}$. The timed liquid injection cycle can be set to any value, but the current set up used provides sufficient pump-down time using $10 \mathrm{sec}$ between injections. Every simulation presented here was performed with this set of parameters.

\subsection{Vaporization kinetic modeling with one species}

At least three differential equations have to be solved sequentially: one for the mass balance of droplets, one for the heat balance of droplets and one for the gas mass balance into the reactor with pumping. In a first approach, the set of equations with just one species will be described. The next section will deal with the case where a mixture of solvent and precursor is evaporated. The first modeling step will help us understand the main parameters playing a role in the droplet vaporization time.

Under vacuum, the atoms escape from the liquid surface at the maximal rate allowed by the surface temperature of the droplet, following a Knudsen-type kinetics of vaporization [12]:

$$
\phi_{\text {vap }}=A_{\text {droplet }} \frac{2}{2-\alpha_{C}} \frac{1}{\sqrt{2 \pi M_{\text {solvent }} R}}\left(\alpha_{C} \frac{P_{\text {reactor }}}{\sqrt{T_{\text {reactor }}}}-\alpha_{E} \frac{P_{\text {sat }\left(T_{\text {droplet }}\right)}}{\sqrt{T_{\text {droplet }}}}\right) M_{\text {solvent }}
$$

The value of $\alpha_{C}, \alpha_{E}$, the condensation and evaporation coefficients, is taken to be about 0.1 for a liquid or solid surface vaporizing into vacuum [13]. Eq. (1) describes a balance between molecules escaping from the droplet surface and molecules returning from the gas phase to the surface. 
A simple mass balance on a droplet can be derived from Eq. (1):

$$
\frac{d m_{d r o p l e t}}{d t}=-\phi_{v a p}
$$

The heat balance on a droplet is written as follows:

$$
m_{\text {droplet }} C p_{\text {droplet }} \frac{d T_{\text {droplet }}}{d t}=-\phi_{\text {vap }} \cdot \Delta H_{\text {vap }}+A_{\text {droplet }} \sigma \varepsilon\left(T_{\text {reactor }}^{4}-T_{d r o p l e t}^{4}\right)
$$

Finally we compute the change of pressure with time in a given reactor volume connected to a vacuum pump:

$$
\frac{V_{\text {reactor }}}{R T_{\text {reactor }}} \frac{d P_{\text {reactor }}}{d t}=\frac{N_{\text {droplets }} \phi_{\text {vap }}}{M_{\text {solvent }}}-\frac{Q_{\text {pump }} P_{\text {reactor }}}{R T_{\text {reactor }}}
$$

The volumetric pumping rate is considered as constant at the exit of the reactor whatever the pressure. Equ. (2) - (4) are solved with a fourth order Runge-Kutta method with a time step of $10^{-7}$ seconds for the two first milliseconds of integration and $10^{-4}$ seconds until the end of calculation. Other solver algorithms with variable time stepping for example lead to huge numerical instabilities. The solution does not depend on the time step with this set of parameters. The solver is stopped for Equ. (2) - (3) when the droplet mass reaches one millionth of its initial mass (or one percent of its initial diameter) to avoid calculation issues at zero diameter. Equ. (4) is then solved with the second right member only until the end of the pulse period.

Figures 1, 2 and 3 present the droplet diameter, the droplet temperature and the reactor vapor pressure in case of release of $0.1 \mathrm{~g}$ of hexane under the case study conditions. The total vaporization time is $1.27 \mathrm{~s}$. The time-axis has been normalized by the pulse time to show the process progression from start to finish, from 0 to 1 .

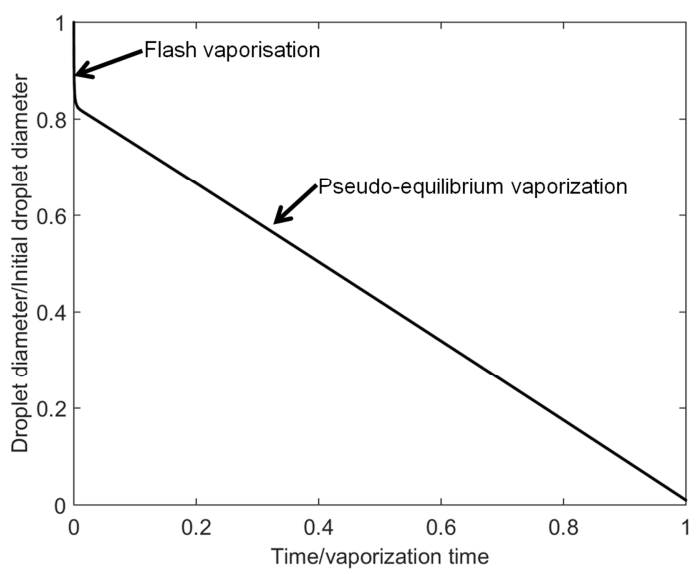

Figure 1: droplet diameter versus time with pure hexane 


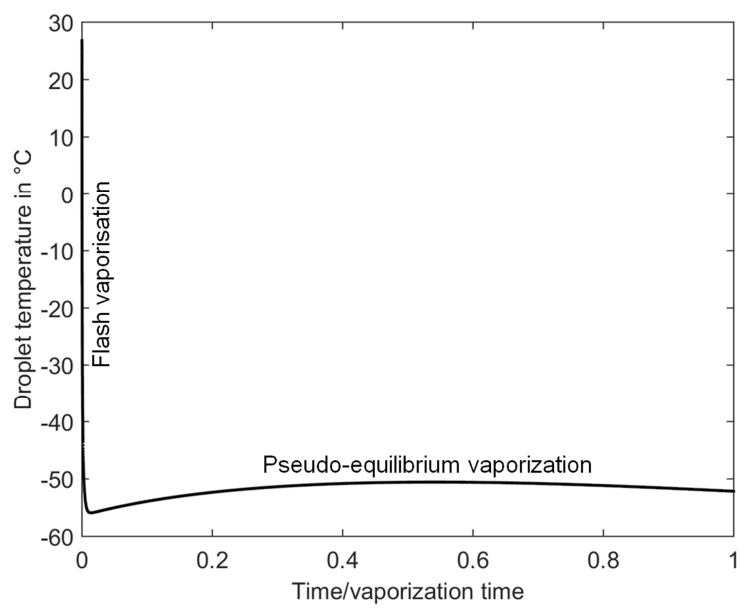

Figure 2: droplet temperature versus time with pure hexane

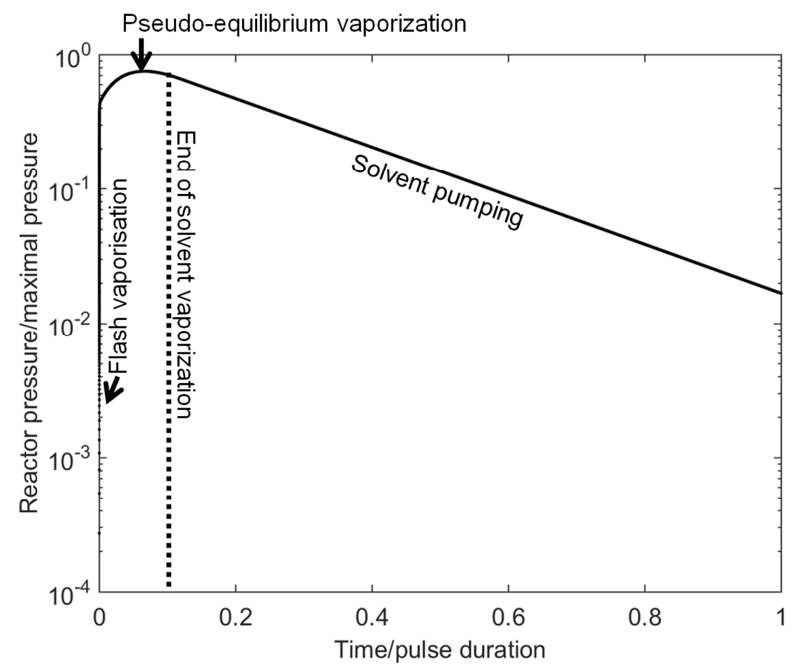

Figure 3: reactor pressure versus time with pure hexane for 10 seconds

The striking result is that under the case study conditions, hexane vaporization is not instantaneous but rather proceeds in two steps, flash vaporization followed by pseudoequilibrium. Flash vaporization corresponds to the first step where the heat of vaporization of a droplet is mainly provided by its own internal enthalpy. The limitation of vaporization kinetic is due to mass transport. Evaporation of molecules cools down the remaining liquid which in turn decreases the vapor pressure. This phenomenon is quick (few milliseconds) so the external radiation does not play a crucial role. Once the temperature of the droplet reaches a point where its vapor pressure equals the reactor pressure, flash evaporation ceases and a pseudo equilibrium-vaporization starts. Pseudo-equilibrium vaporization is the step where the only remaining heat source for phase change is the external radiation flux from the surroundings in the reactor. Heat transfer to the drop imposes the limitation on vaporization kinetics. Pseudo-equilibrium vaporization continues until complete vaporization of the liquid phase. We assume that due to the absence of critical nuclei in the high-purity solvent droplets, boiling phenomena is unlikely and the droplets keep their spherical shape during the whole process. 


\subsection{Sensitivity analysis}

Vaporization efficiency is defined as the percentage of liquid vaporized. Un-vaporized liquid drops can impinge on the reactor walls and remain in a liquid state if the reactor walls are at a low enough temperature. The parameter that most directly affects the vaporization efficiency is the lifetime of droplets, which determines the time of flight. A short lifetime releases the precursor vapor in a sudden sharp pulse, and the pressure peak in the reactor is higher. Thus, evaporation with a short lifetime should produce the conditions for the highest film deposition rate and the most uniform deposition. Figure 4 presents the sensitivity analysis on the vaporization time of pure hexane droplets released at zero pressure for the case study conditions $( \pm 10 \%$ of every parameter of the case study conditions, except properties of hexane, which are kept constant).

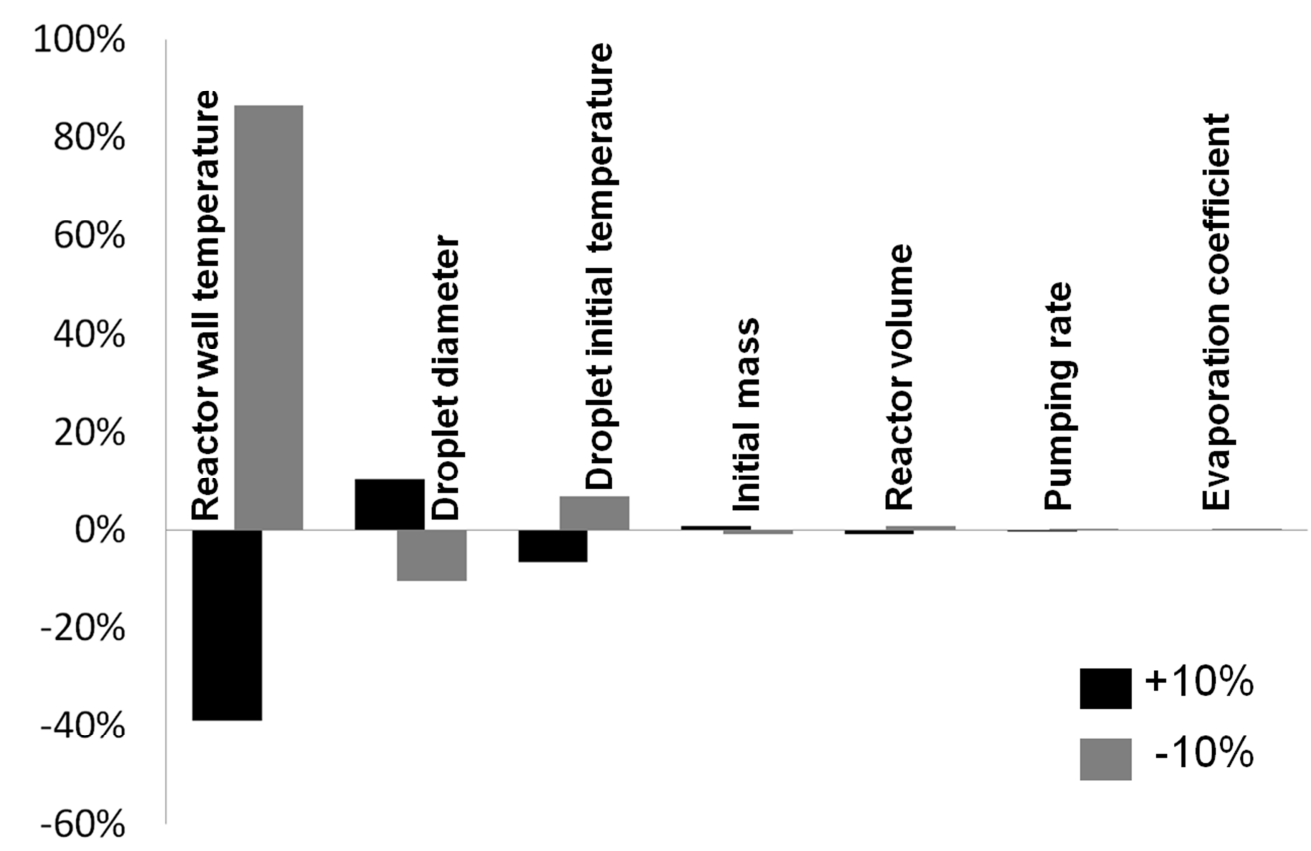

Figure 4: sensitivity analysis of different parameters on the vaporization time of hexane: effect on a $\pm 10 \%$ variation of parameter on vaporization time.

The vaporization time is insensitive to the assumptions of condensation and vaporization coefficients values $(\approx 1 / 100$ ratio). Vaporization time is also relatively insensitive to pumping rate $(\approx 1 / 50)$ and reactor volume $(\approx 1 / 10)$, as well as to the mass injected in one pulse $(\approx 1 / 10)$. Previously reported research for deposition on nano-scale trenches validates this finding, as the pulse timing and injection volume were not found to affect conformal deposition [14]. The most important parameter controlling the vaporization time, apart from the droplet diameter $(\approx 1 / 1)$ and droplet initial temperature $(\approx 1 / 1)$, is the reactor wall temperature $(\approx 5 / 1)$. The slower pseudo-equilibrium evaporation step relies on a radiation balance only. It is worth noting that increasing the initial enthalpy of liquid phase by heating the precursor solution is not as effective as heating the reactor walls to decrease vaporization time.

The results of the simple vaporization model inform the design and operation of the pp-CVD technology. Atomization to small droplets is key, pre-heating the solution can assist in 
achieving the flash evaporation, but a heated reactor wall is the key to high vaporization efficiency. Obviously, the reactor wall temperature needs to be below the decomposition temperature of the precursor, or the overall deposition conversion efficiency will be reduced by parasitic wall deposition.

\subsection{Vaporization kinetic modeling with two species}

The influence of the choice of the liquid solvent/precursor is analyzed using a similar approach. A liquid mixture containing a precursor plus a solvent is nearly always required to reduce the viscosity sufficiently for atomization to small droplets. The analysis of the problem is obviously more complicated. Raoult's law for mixtures is applied. The kinetics of vaporization according to the Labuntsov model as given in Eq. (1) is applied for each species, using their respective partial pressures, and multiplied by their respective mole fraction in the liquid phase.

$$
\begin{aligned}
& \phi_{\text {vap,solvent }}=\phi_{\text {vap }, \text { solvent,Labuntsov }} \cdot x_{\text {solvent }} \text { with } x_{\text {solvent }}=\frac{m_{\text {solvent }} / M_{\text {solvent }}}{\frac{m_{\text {solvent }}}{M_{\text {solvent }}}+\frac{m_{\text {precursor }}}{M_{\text {precursor }}}} \\
& \phi_{\text {vap,precursor }}=\phi_{\text {vap } \text {, precursor,Labuntsov }} \cdot\left(1-x_{\text {solvent }}\right)
\end{aligned}
$$

The two mass balances on a droplet become:

$$
\begin{aligned}
& \frac{d m_{\text {solvent }}}{d t}=-\phi_{\text {vap,solvent }} \\
& \frac{d m_{\text {precursor }}}{d t}=-\phi_{\text {vap,precursor }} \\
& m_{\text {droplet }}=m_{\text {solvent }}+m_{\text {precursor }}
\end{aligned}
$$

The heat balance becomes:

$$
\begin{aligned}
& m_{\text {droplet }} C p_{\text {mixture }} \frac{d T_{\text {droplet }}}{d t}=-\phi_{\text {vap, solvent }} . \Delta H_{\text {vap, solvent }}-\phi_{\text {vap }, \text { precursor }} . \Delta H_{\text {vap }, \text { precursor }}+A_{\text {droplet }} \sigma \varepsilon\left(T_{\text {reactor }}^{4}-T_{\text {droplet }}^{4}\right) \\
& \text { With } C p_{\text {mixture }}=\frac{C p_{\text {solvent }} m_{\text {solvent }}+C p_{\text {precursor }} m_{\text {precursor }}}{m_{\text {solvent }}+m_{\text {precursor }}}(11)
\end{aligned}
$$

And finally the partial pressure balances become:

$$
\begin{aligned}
& \frac{V_{\text {reactor }}}{R T_{\text {reactor }}} \frac{d P_{\text {solvent }}}{d t}=\frac{N_{\text {droplets }} \phi_{\text {vap }, \text { solvent }}}{M_{\text {solvent }}}-\frac{Q_{\text {pump }} P_{\text {solvent }}}{R T_{\text {reactor }}} \\
& \frac{V_{\text {reactor }}}{R T_{\text {reactor }}} \frac{d P_{\text {precursor }}}{d t}=\frac{N_{\text {droplets }} \phi_{\text {vap, precursor }}}{M_{\text {precursor }}}-\frac{Q_{\text {pump }} P_{\text {precursor }}}{R T_{\text {reactor }}} \\
& P_{\text {reactor }}=P_{\text {solvent }}+P_{\text {precursor }} \quad(14)
\end{aligned}
$$


Solving this set of equations is carried out using the same approach as for the single species.

Figures 5, 6 and 7 present the droplet diameter, the droplet temperature and the reactor vapor pressure in case of release of $0.1 \mathrm{~g}$ of a 10:1 mass ratio hexane/TTIP mixture using the case study conditions. The total vaporization time is found to be $2.35 \mathrm{~s}$, nearly two times longer than for pure hexane only.

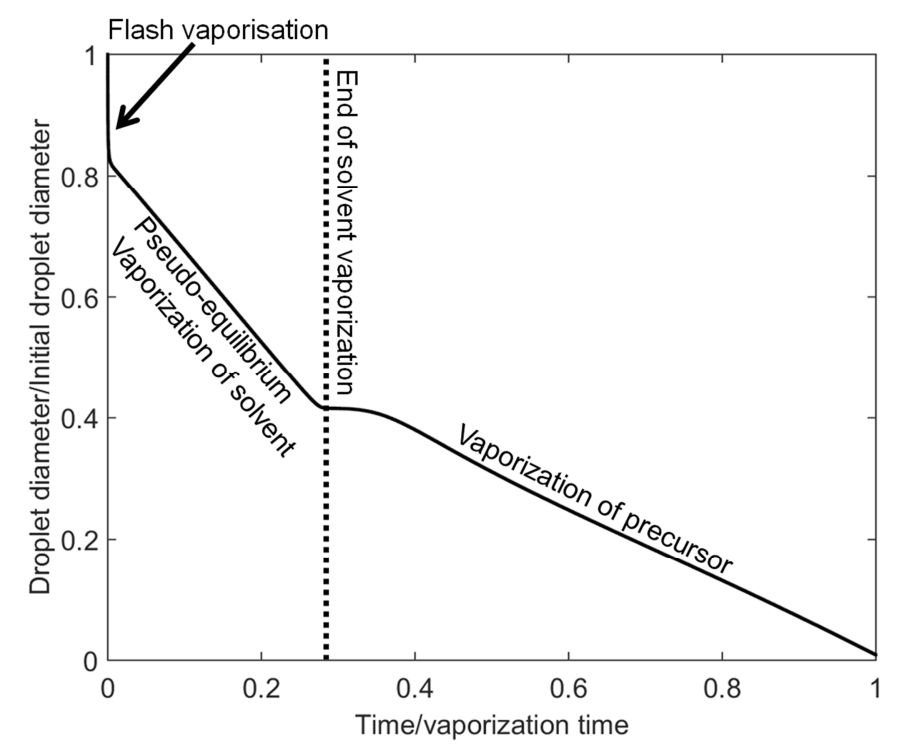

Figure 5: droplet diameter versus time with 10:1 hexane/TTIP mixture

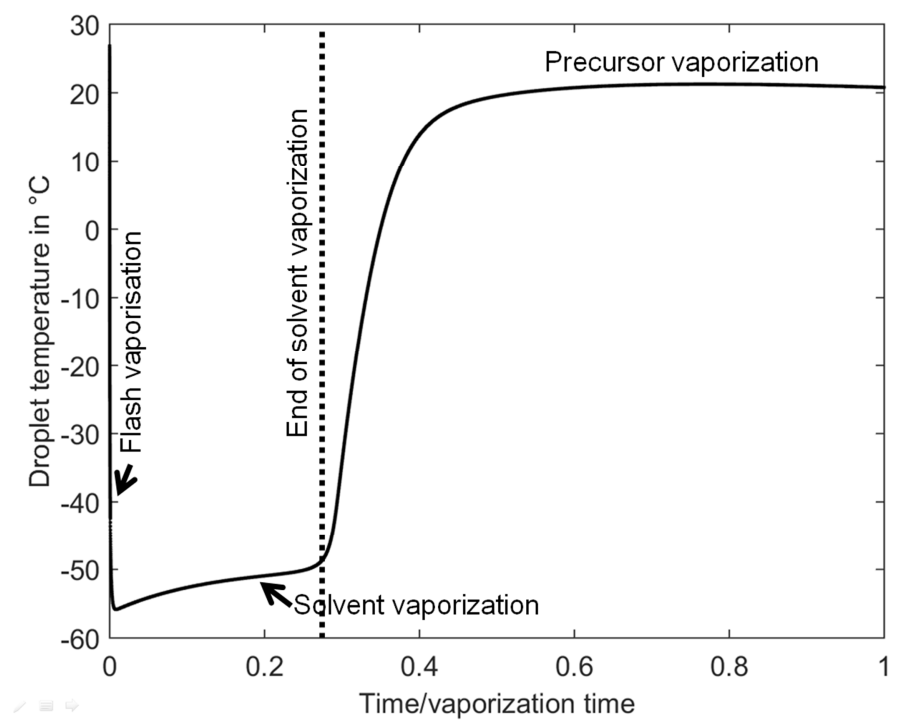

Figure 6: droplet temperature versus time with 10:1 hexane/TTIP mixture 


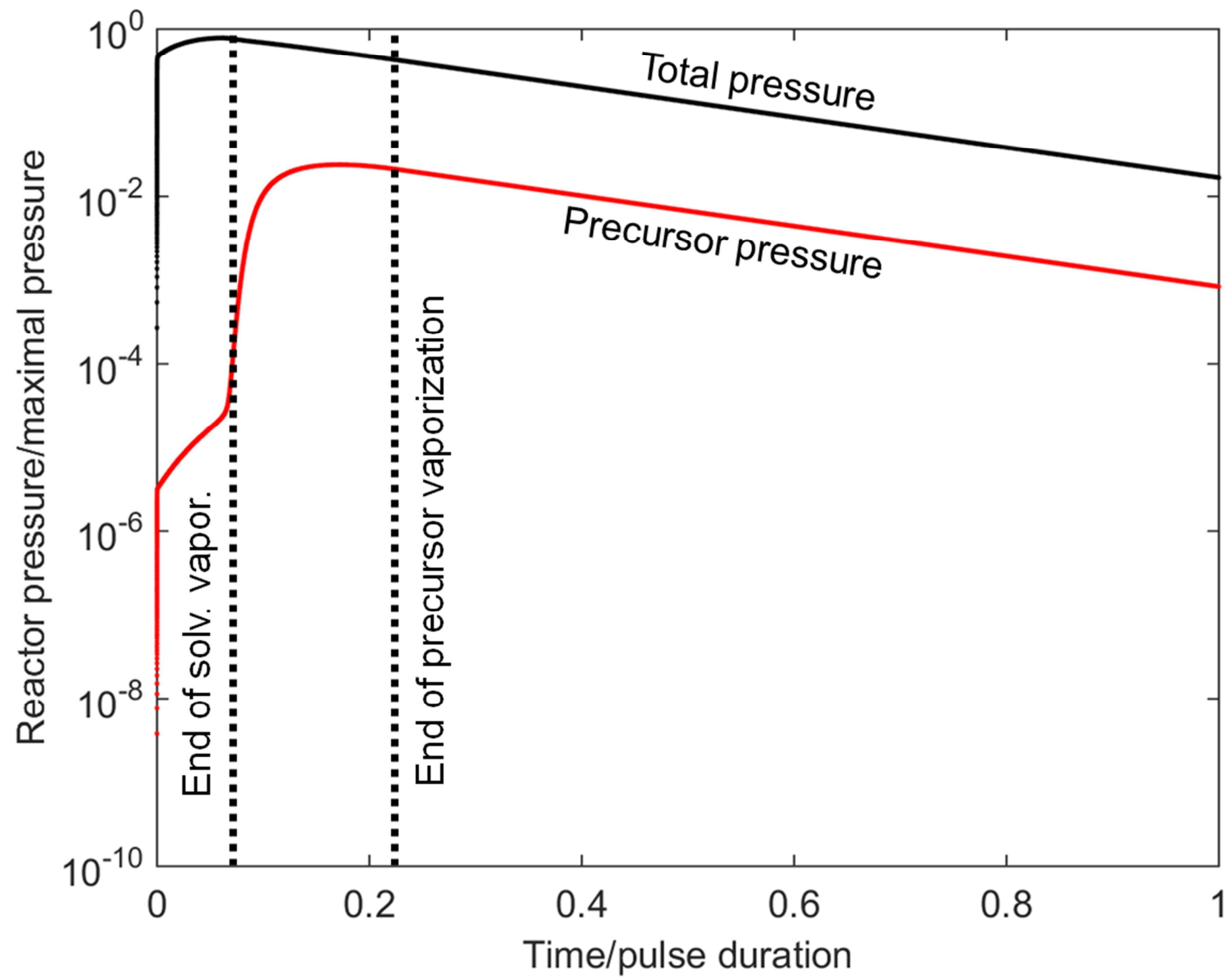

Figure 7: reactor pressure versus time with 10:1 hexane/TTIP mixture for 10 seconds.

On figure 7, pressure is normalized by the pressure calculated if the whole liquid mass (TTIP + hexane) is instantaneously set to gas phase at the reactor wall temperature (300 K).

The mixture hexane/TTIP is chosen due to the fact that these two liquids present contrasted properties. Hexane requires huge heat input to vaporize as the enthalpy of evaporation is $\Delta \mathrm{H}_{\text {vap }}=334 \mathrm{~kJ} / \mathrm{kg}$ in spite of its high vapor pressure, $P_{\text {sat }}=21819 \mathrm{~Pa}$ at $300 \mathrm{~K}$. TTIP is thermodynamically much easier to evaporate at room temperature with $\Delta \mathrm{H}_{\text {vap }}=62.3 \mathrm{~kJ} / \mathrm{kg}$ but presents a very low vapor pressure, $P_{\text {sat }}=16.51 \mathrm{~Pa}$ at $300 \mathrm{~K}$. During the droplet evaporation process, the hexane evaporates first and the droplet temperature is reduced to the point at which the TTIP vapor pressure becomes negligible. The peak pressure of TTIP is consequently delayed compared to the main peak pressure, the precursor vapor may not benefit as greatly from the volume expansion process, and may be impeded to some degree by diffusion through the solvent vapor which would already be present in the reactor. It should be noted, however, that there is no inlet flow and the continuous pumping down means that there is no bulk vapor viscous flow pattern in the reactor through which the TTIP vapor would diffuse as is found in carrier gas CVD mass transport.

After the hexane solvent is evaporated, the droplet temperature rises through radiative heating to a new plateau where pure TTIP remains and evaporates. We think this plateau at temperature close to the reactor wall is typical of deposition kinetics limited by mass transport. Using hexane as the solvent would thus not be ideal for high TTIP evaporation 
efficiency prior to reaching the substrate or reactor walls. It appears clearly here that hexane used as solvent retards the vaporization TTIP.

\subsection{Tracking the droplets}

Another interesting parameter to calculate apart from the vaporization time is the vertical position of droplets if the vaporization nozzle is at the top of a vertical plug flow reactor with a cylindrical shape. In this particular case, as the volume pumping rate of a vacuum pump is nearly constant with pressure, the vertical axial velocity of gases is also constant with pressure and depends on the pumping rate only. The axial velocity of gas into the reactor from the vaporization zone at the top is simply given by:

In the free molecular regime, the force balance on a droplet is given by:

$$
\begin{gathered}
v_{\text {reactor }}=Q_{\text {pump }} / S_{\text {reactor }} \\
\frac{d z_{\text {droplet }}}{d t}=v_{\text {droplet }}(16) \\
\frac{d v_{\text {droplet }}}{d t}=\frac{\frac{3 \pi \eta_{\text {gas }} d_{\text {droplet }}}{C_{c}}\left(v_{\text {reactor }}-v_{\text {droplet }}\right)\left(1+0.15 \mathrm{Re}_{\text {droplet }}^{0.687}\right)}{m_{\text {droplet }}}-g
\end{gathered}
$$

The viscous drag force is corrected by the Cunningham correction factor, which depends on the Knudsen number. The relative signs correspond to a case where the gravity force vector is in the same direction as the velocity vector of gas (top-down direction). The surrounding gas is assumed to be mainly solvent molecules for simplification:

$$
\rho_{\text {gas }}=\frac{P_{\text {reactor }} M_{\text {solvent }}}{R T_{\text {reactor }}}
$$

The droplet Reynolds number is given by:

$$
\operatorname{Re}_{\text {droplet }}=\frac{\rho_{\text {gas }}\left|v_{\text {droplet }}-v_{\text {reactor }}\right| d_{\text {droplet }}}{\eta_{\text {gas }}}
$$

The Cunningham correction factor is given by: 


$$
\begin{aligned}
& C_{c}=1+\frac{2 l_{p m}}{d_{\text {droplet }}}\left(1.257+0.4 \exp ^{-0.55 \frac{d_{\text {droplet }}}{l_{p m}}}\right) \\
& l_{p m}=\frac{1}{\sqrt{2} \pi \sigma_{\text {solvent }}^{2} c}(21) \\
& c=\frac{N_{a} P_{\text {reactor }}}{R T_{\text {reactor }}}(22)
\end{aligned}
$$

Finally, the viscosity has to be calculated according to the Enskog theory [15]:

$$
\begin{aligned}
& \eta_{\text {gas }}=2,6693.10^{-6} \frac{\sqrt{M_{\text {solvent }} T_{\text {reactor }}}}{\sigma_{\text {solvent }}^{2} \Omega_{\eta}} \\
& T^{*}=\frac{k_{b} T_{\text {reactor }}}{\varepsilon_{\text {solvent }}} \\
& \Omega_{\eta}=\frac{1.16145}{T^{* 0.14874}}+\frac{0.52487}{\exp \left(0.7732 T^{*}\right)}+\frac{2.16178}{\exp \left(2.43787 T^{*}\right)}
\end{aligned}
$$

The Enskog theory for mixtures [15] is used to compute the viscosity of gas if the precursor mole fraction is not considered negligible. This set of equations is solved with the same integration parameters and method used for Eq. (2)-(4) simultaneously without any particular difficulty. Figure 8 presents the droplet vertical velocity versus time of hexane under the case study conditions. The total vaporization time is still $1.27 \mathrm{~s}$.

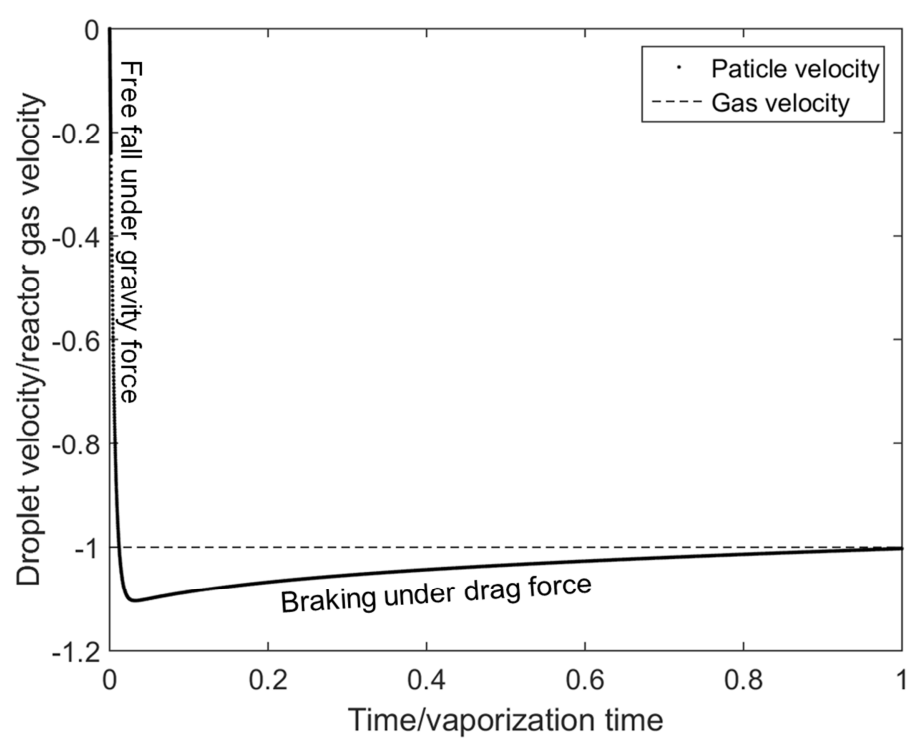

Figure 8: Vertical velocity of a $18 \mu$ m hexane droplet evaporating completely. 
It appears that for micron-size droplets, the main force acting on particles is the drag force. The distance travelled during the free-fall flight is negligible compared to the drag flight. In the case presented here, the particles would readily be entrained in any gas bulk flow. This point is important because it means also that the initial momentum of a particle due to the spray generator would also rapidly be absorbed by drag, within a fraction of a second after being released by the spray nozzle if the reactor vapor is quiescent. In our previous experimental and flow modeling research, we have demonstrated that the pulsed vaporization operation does not produce a bulk flow condition unless the vaporization zone is isolated from the reactor volume by an orifice or constriction $[18,19]$.

\section{Optimal process parameters and control}

The models are used to explore optimal design and operating conditions to achieve the highest possible vaporization efficiency and the most rapid pressure rise. The liquid vaporization time must be as short as possible for two reasons. Fast evaporation allows liquid droplets to vaporize prior to reaching the reactor wall or the deposition surface in order to avoid splats and non-uniform deposition. A sharp rise in peak pressure, as intense as possible, ensures strong pressure variations into the whole reactor volume for a short time period, which drives mass transport by gas expansion. These optimal processes are obviously intrinsically connected through the processing objectives.

- The vaporization efficiency must be maximized. Un-vaporized precursor is most likely to stick or react on the reactor walls or exhaust lines and be lost to the deposition process.

- The pressure peaks of solvent and precursor must be concomitant in order to ensure control of the precursor supply and uniformity of mass transport.

- The throughput of the reactor should be high. The maximal amount of precursor must be inserted at each liquid pulse in order to provide the highest growth rate while maintaining deposition uniformity.

Two process design routes can be explored to achieve the optimal processing objectives, the reactor hardware or the physical chemistry of the reactants.

\subsection{Process Optimization by Hardware Selection}

The sensitivity analysis presented in figure 4 clearly highlights that the main parameter to control is the wall temperature to provide the heat to droplets for vaporization during the quasi-equilibrium vaporization phase. Of lesser importance is the particle size and initial liquid temperature for a given precursor/solvent couple. The liquid vaporization process requires good atomization, but is not sensitive to the reactor volume or the pumping system. Injecting more mixture at each pulse does not impede much the vaporization time, so this quantity can be increased easily.

\subsection{Process Optimization by Solvent Selection}

As an illustration, we will compare hexane and toluene as the solvent. This may seem counter intuitive to engineers when the objective is rapid evaporation. Hexane is a much more volatile solvent than toluene, with $P_{\text {sat }}=21819 \mathrm{~Pa}$ for hexane compared to $P_{\text {sat }}=4171 \mathrm{~Pa}$ for toluene at $300 \mathrm{~K}$. But the high evaporation enthalpy of hexane leads to a strong cooling of droplets, 
probable concentration of the solution with more TTIP in the pseudo-equilibrium stage. The vaporization efficiency seems to be much lower for hexane than for toluene [16]. Previous studies with toluene and TTIP have also demonstrated that very high vaporization efficiency is possible [17], and another solvent has not been investigated experimentally. However, a recent study in our group comparing hexane and toluene solvent for various alumina precursors did find that vaporization was particularly poor when hexane was used [16].

In the flash vaporization step, the ratio $\Delta \mathrm{H}_{v a p} / \mathrm{C}_{p}$ must be as low as possible in order to maximize the instantaneously flashed mass, resulting in the steepest rise in pressure and highest peak pressure in the reactor. However, the sensitivity analysis proves that the initially stored enthalpy is not so effective in reducing vaporization time.

In the pseudo-equilibrium vaporization step, the heat balance indicates us that the heat capacity of solvent/precursor plays nearly no role (constant temperature step), only the capacity to generate vapor from liquid with the minimal amount of energy plays a role. The precursor with the minimal heat of vaporization $\Delta \mathrm{H}_{v a p}$ will provide the optimal vaporization efficiency and ensure a fast vaporization for any droplets remaining after the initial flash step.

Finally, the solvent and precursor should evaporate at similar rates so that the expansion process for precursor and solvent vapors are concomitant. Their vapor pressure must therefore be as close as possible, whatever the temperature.

The influence of each parameter is investigated by performing a sensitivity analysis on the properties of hexane droplets in the case study conditions used by varying the heat of vaporization $(334 \mathrm{~kJ} / \mathrm{kg} \pm 10 \%)$, the heat capacity $\left(2293 \mathrm{~J} \mathrm{~kg}^{-1} \cdot \mathrm{K}^{-1} \pm 10 \%\right)$ and the vapor pressure $\left(P_{\text {sat }} \pm 10 \%\right.$, depending on temperature) and calculating the vaporization time. Figure 9 presents the sensitivity analysis results.

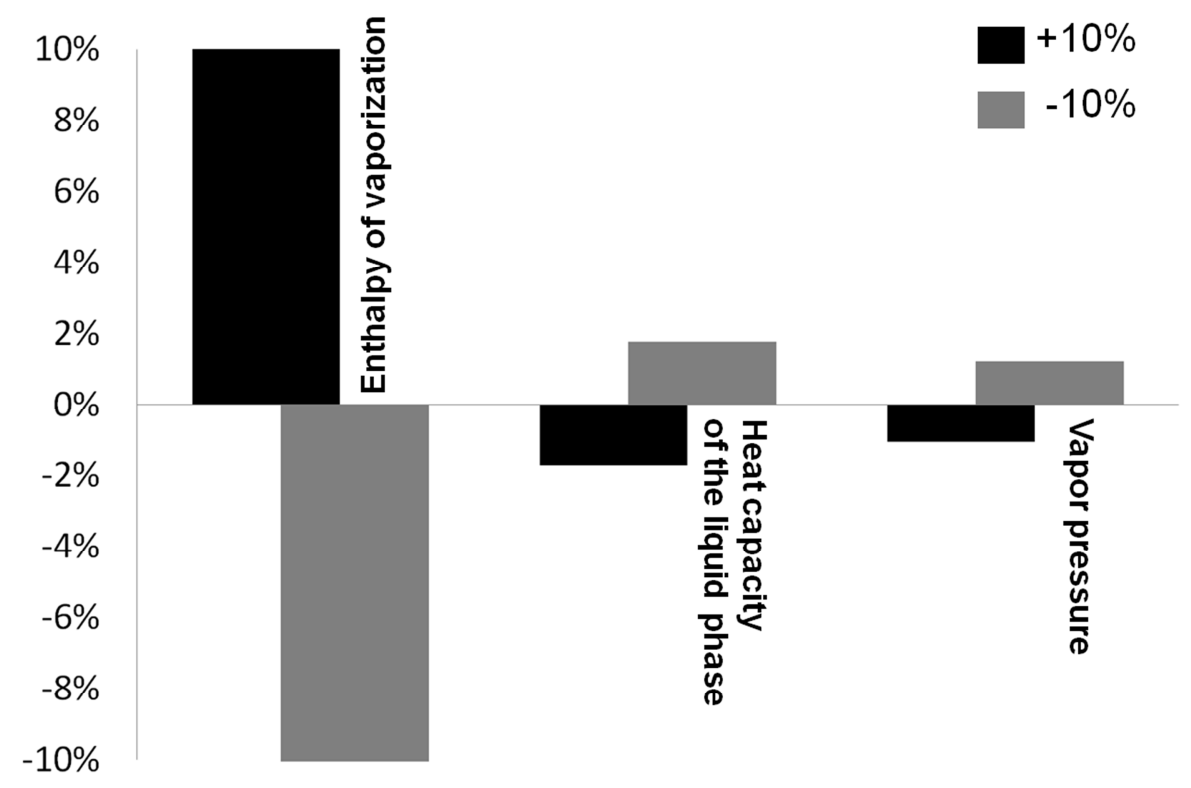

Figure 9: sensitivity analysis of the properties of hexane: effect on a $\pm 10 \%$ variation of parameter on vaporization time

Clearly the enthalpy of vaporization is a key parameter to shorten the vaporization time $(\approx 1 / 1$ ratio). The influence of vapor pressure is strikingly secondary $(\approx 1 / 10)$. This can be explained 
by the fact that under the pseudo-equilibrium vaporization, the slowest part of the process, the evaporation mass flux is mainly limited by phase change. Vapor pressure plays a role only in the kinetics of the flash vaporization step, which is very short. Finally the influence of liquid heat capacity $(\approx 1 / 5)$ on the vaporization time is rather low for the same reason.

These assumptions are assessed by simulating the vaporization story of $0.1 \mathrm{~g}$ of a 10:1 mass concentration toluene/TTIP mixture for a 10 seconds pulse time under the case study conditions. Figure 10 presents the pressure versus time chart. Figure 11 presents the droplet temperature with time. The total vaporization time is $1.89 \mathrm{~s}$, which is shorter than for the mixture with hexane.

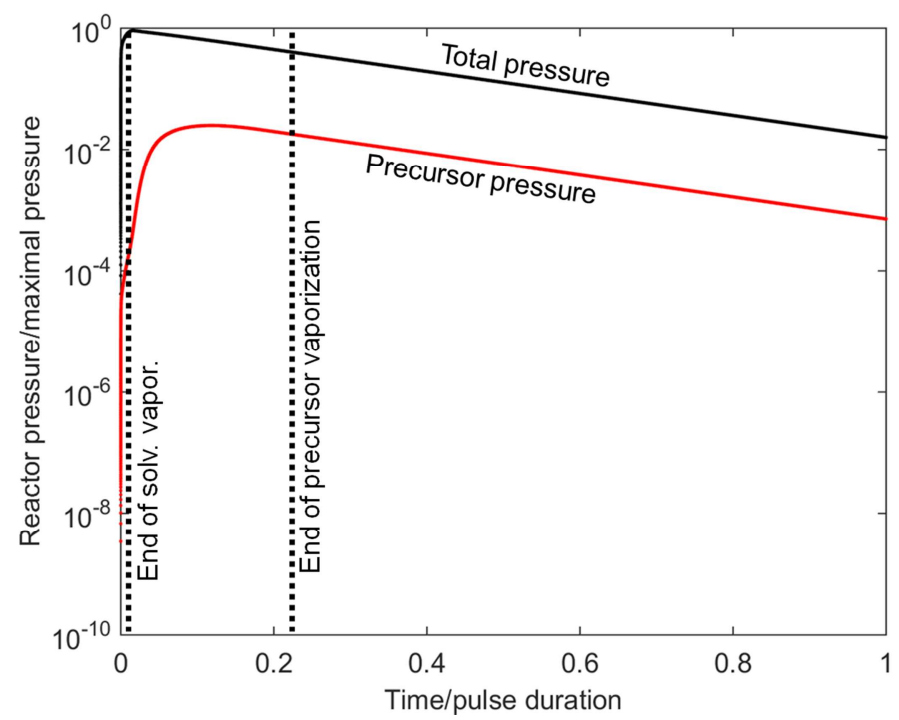

Figure 10: reactor pressure versus time with 10:1 toluene/TTIP mixture for 10 seconds.

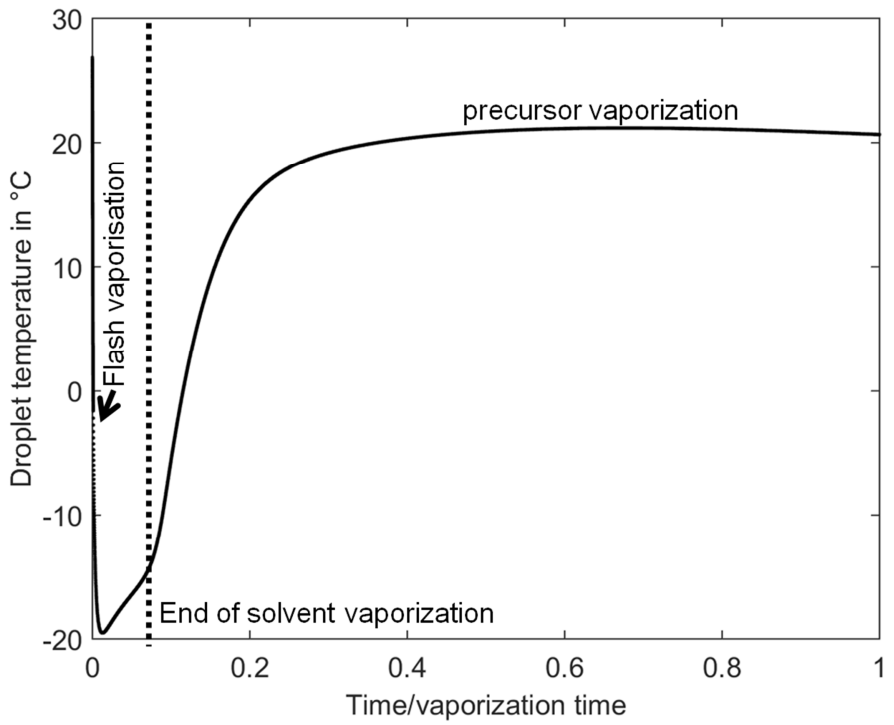

Figure 11: droplet temperature versus time with 10:1 toluene/TTIP mixture.

On figure 10, pressure is divided by the pressure calculated if the whole liquid mass (TTIP + toluene) is instantaneously set to gas phase at reactor wall temperature $(300 \mathrm{~K})$. 
Toluene has a $\Delta \mathrm{H}_{\text {vap }}$ of $38.1 \mathrm{~kJ} / \mathrm{kg}$ at $298 \mathrm{~K}$ while the $\Delta \mathrm{H}_{\text {vap }}$ of TTIP is about of $62.3 \mathrm{~kJ} / \mathrm{kg}$ at $298 \mathrm{~K}$. These two values are very similar and relatively low. The $\mathrm{P}_{\text {sat(toluene) }} / \mathrm{P}_{\text {sat(TTIP) }}$ is about 250 whatever the temperature in the calculation range and is low compared to hexane/TTIP couple, which is about 1300 in the calculation range. Toluene and TTIP still evaporate at different rates but without undergoing strong undercooling due to their low heat of vaporization. The droplet equilibrium temperature stays above $-20^{\circ} \mathrm{C}$ compare to the $-50^{\circ} \mathrm{C}$ of the hexane/TTIP couple (see Fig. 6). The total peak pressure is sharp and instantaneous compared to the pulse duration. The peak pressure of TTIP appears early compared to the one with hexane mixture but its sharpness is the same. In the case study presented here, hexane acts as a retarding compound for TTIP vaporization due to its high enthalpy of vaporization. TTIP, due to its very low vapor pressure compared to solvents, has a low vaporization kinetic impeding the vaporization time of droplets. The plateau in temperature close to the reactor wall temperature still indicates a vaporization kinetic controlled by mass transport. A sensivity analysis on pure TTIP droplets under the case study conditions shows a low influence of its enthalpy of vaporization $(\approx 1 / 10$ ratio) with a moderate influence of its vapor pressure $(1 / 1$ ratio) on the vaporization time, confirming this hypothesis.

From these results we can formulate a rule of thumb for precursor/solvent choice in pulsed CVD: the precursor and solvent must have low enthalpy of vaporization and high vapor pressures which are also as similar as possible.

Of course, the finite number of solvents and precursors, their relative viscosities and cosolubility limits strongly the choice of available couples and mixtures. The purpose of the numerical modeling is to understand the complex phenomena and provide design trends where a choice of solvents and precursors is possible. Using the model can eliminate many weeks of experimental trial and error if the chemical physical properties are available.

\subsection{Process Optimization by Shape Reactor Design}

In conventional steady flow CVD processes the reactor geometry has a profound influence on the uniformity and efficiency. Previous detailed numerical modeling of the expansion mass transport process indicated that a large open chamber would provide the most uniform wellmixed reactor conditions [18]. Experimental results have provided observations in line with the flow modeling studies [19]. We propose to assess the liquid pulse CVD process by the means of two non-dimensional parameters, depending on the fact that the reactor shape is close or far from a long tube (plug type reactor or unspecified vessel).

We assume the vertical tube reactor would have the nozzle at the top, the susceptor at the bottom, and the vacuum evacuation in the end zone of the tube beyond the substrate location. In this configuration liquid droplets can impinge on the reactor walls and could reach the susceptor. Depending on the vaporization process and the thermodynamic properties of the precursor, one of two possibilities could arise. The radiant heat of the susceptor could provide the vaporization energy and complete the vaporization of any droplets that come near the substrate, resulting in smooth, vapor-deposited films. If the heat from the susceptor only provides the heat to vaporize the solvent, but the precursor requires much more energy to vaporize, then a precursor aerosol may arrive at the surface and subsequently be decomposed, resulting a lumpy and possibly amorphous films.

Comparing the falling distance to the nozzle-susceptor distance is relevant. The process operates optimally if the droplet vaporizes completely before reaching the substrate: 


$$
\frac{l_{\text {vaporization }}}{l_{\text {nozzle-substrate }}}<<1
$$

Note that in case of horizontal tube reactor, Eq. (16)-(17) would need to be modified. We have operated the reactor in ways that guarantee aerosol production to study the trajectory of particles under the unique injection and pump-down conditions. The observations are clear that aerosols generated during the evaporation phase where the vapors are expanding are uniformly ejected in all directions away from the nozzle, but that they also travel in a direct line of site flight path once they are formed. Note that the continuous evacuation of the reactor, the continuously decreasing pressure, disrupts development of continuum flow patterns [20].

If the reactor has an unspecified shape or if the substrate is placed in the chamber not in the line of sight aerosol flight path, comparing the time for complete vaporization to the residence time of gas into the chamber is relevant. The process may operate optimally if:

$$
\frac{\tau_{\text {vaporization }}}{\tau_{\text {reactor }}}<<1 \text { with } \tau_{\text {reactor }}=\frac{V_{\text {reactor }}}{Q_{\text {pump }}}
$$

This means that the droplets have a small probability to exit the reactor or touch any solid surface without being evaporated. Of course, contrary to a vertical tube-type reactor, each reactor should present its own optimal ratio window.

It will not likely be possible to model the flight path length or time exactly due to lack of property data for the precursors. However, this modeling indicates a process development method that could be used to design the provision of heat for complete vaporization. The reactor walls can be examined to determine the extent of precursor aerosol impingement. Firstly, the susceptor can be located out of the line of sight of the aerosols by placing a shield between the nozzle and the substrate to catch any aerosols. Secondly, the walls of the reactor can be warmed by external heating to provide the droplets heat of vaporization. This was in fact successfully trialed and reported by Lee et al. [19] for TTIP/toluene with the injection operated to purposefully produce aerosols.

\section{Conclusion}

The evaporation kinetics of liquid droplets into a vacuum was found to be primarily controlled by thermodynamics and heat transfer. The numerical modeling of pulsed CVD is a simple way to study the influence of choice of solvent and reactor design to achieve optimal vaporization efficiency and well-mixed reactor conditions necessary for deposition uniformity. It was found that providing radiant heating in the reactor zone is the key parameter for the quick vaporization of liquid precursors. Then a precursor/solvent mixture with a low heat of vaporization and high vapor pressure must be chosen. Finally the precursor/solvent mixture sprayed under the form of small and hot droplets is desirable. The details of the heat transfer around the susceptor and reactor design were not included in this OD modeling and left for future work with a specific reactor for experimental validation. The 
behavior of each precursor/solvent candidate system must ultimately be investigated experimentally. But this model provides understanding of the influence of the relevant design parameters. Numerical modeling and experimental investigation of a delivery system using pulsed injection of metalorganic precursors directly under the gas phase rather than liquid phase is an area of interest for future work.

\section{Acknowledgements}

The authors want to thank Grenoble-INP for the visiting professor program 2014 allowing this study.

\section{Appendix :}

\begin{tabular}{|c|c|c|}
\hline$A_{\text {droplet }}$ & Surface area of a droplet at a given time & $\mathrm{m}^{2}$ \\
\hline$C c$ & Cunningham correction factor & - \\
\hline$C p_{\text {droplet }}$ & Heat capacity of the droplet & $\mathrm{J} \cdot \mathrm{kg}^{-1} \cdot \mathrm{K}^{-1}$ \\
\hline$d_{\text {droplet }}$ & droplet diameter & $\mathrm{m}$ \\
\hline$g$ & Gravity acceleration constant & $\mathrm{m} \cdot \mathrm{s}^{-2}$ \\
\hline$k_{b}$ & Boltzmann constant & $\mathrm{m}^{2} \mathrm{~kg} \mathrm{~s}^{-2} \mathrm{~K}^{-1}$ \\
\hline$l_{\text {vaporization }}$ & Vertical falling distance until complete vaporization & $\mathrm{m}$ \\
\hline$l_{\text {nozzle-substrate }}$ & Nozzle to substrate distance & $\mathrm{m}$ \\
\hline$l_{p m}$ & Mean free path of molecules & $\mathrm{m}$ \\
\hline$m_{\text {droplet }}$ & One droplet mass & $\mathrm{kg}$ \\
\hline$M$ & Molar mass of species & $\mathrm{kg} \cdot \mathrm{mol}^{-1}$ \\
\hline$N_{a}$ & Avogadro number & $\mathrm{mol}^{-1}$ \\
\hline$N_{\text {droplets }}$ & Total number of droplets involved in one pulse & - \\
\hline$P_{\text {reactor }}$ & Time dependent pressure into reactor volume & $\mathrm{Pa}$ \\
\hline$P_{\text {sat }\left(T_{\text {droplet }}\right)}$ & Vapor pressure of species at the droplet temperature & $\mathrm{Pa}$ \\
\hline$Q_{\text {pump }}$ & Vacuum system pumping volume flow rate & $\mathrm{m}^{3} \cdot \mathrm{s}^{-1}$ \\
\hline$R$ & Gas constant & $\mathrm{J} \cdot \mathrm{mol}^{-1} \cdot \mathrm{K}^{-1}$ \\
\hline $\operatorname{Re}_{\text {droplet }}$ & Local droplet Reynolds number & - \\
\hline$S_{\text {reactor }}$ & Surface cross-section of a tube reactor & $\mathrm{m}^{2}$ \\
\hline$T_{\text {droplet }}$ & Droplet temperature (liquid phase) & $\mathrm{K}$ \\
\hline$T_{\text {reactor }}$ & Reactor wall temperature & $\mathrm{K}$ \\
\hline$T^{*}$ & Reduced temperature & - \\
\hline$v_{\text {droplet }}$ & Vertical velocity of a droplet & $\mathrm{m} \cdot \mathrm{s}^{-1}$ \\
\hline$v_{\text {reactor }}$ & Vertical velocity of gas in a tube reactor & $\mathrm{m} \cdot \mathrm{s}^{-1}$ \\
\hline$V_{\text {reactor }}$ & Reactor volume & $\mathrm{m}^{3}$ \\
\hline$x$ & Mole fraction of a species in liquid phase & - \\
\hline$z_{\text {droplet }}$ & Vertical position of a droplet & $\mathrm{m}$ \\
\hline$\alpha_{C}, \alpha_{E}$ & Evaporation and condensation coefficient of species & - \\
\hline
\end{tabular}




$\begin{array}{lll}\Delta H_{\text {vap }} & \text { Enthalpy of vaporization of a given species } & \mathrm{J} . \mathrm{kg}^{-1} \\ \varepsilon & \text { Emissivity } & - \\ \varepsilon_{\text {solvant }} / k_{b} & \text { Lennard-Jones potential well } & \mathrm{K} \\ \eta_{\text {gas }} & \text { Gas viscosity into the reactor } & \mathrm{Pa} . \mathrm{s} \\ \phi_{\text {vap }} & \text { Mass vaporization flux } & \mathrm{kg} \cdot \mathrm{m}^{-2} \cdot \mathrm{s}^{-1} \\ \Omega_{\eta} & \text { Collision integral for viscosity } & - \\ \sigma & \text { Stephan Boltzmann constant } & \mathrm{W} \cdot \mathrm{m}^{-2} \cdot \mathrm{K}^{-4} \\ \sigma_{\text {solvent }} & \text { Lennard-Jones inter-particle distance } & \AA \\ \tau_{\text {reactor }} & \text { Residence time of gas phase into reactor } & \mathrm{s} \\ \tau_{\text {vaporization }} & \text { Time for complete vaporization } & \mathrm{S}\end{array}$

\section{References}

[1] A. C. Jones und M. L. Hitchman, Chemical Vapour Deposition : Precursors, Processes and Applications. Royal Society of Chemistry Publishing, Cambridge, UK, 2009.

[2] S. Krumdieck, and R. Raj. Chemical Vapor Deposition 7, No. 2, 2001, 85-90.

[3] S. Krumdieck, P. Hadley, M. Cave, S. Baluti, M. Jermy, A. Peled. Chemical Engineering Science 62, 2007, 6121-6128.

[4] H. M. Cave, S. Krumdieck, and M. C. Jermy. Chemical Engineering Journal 135, 1-2, 2008, $120-128$.

[5] S. Krumdieck. Acta Materialia 49, 2001, 583-588.

[6] K. L. Choy, Progress in Materials Science 48, 2003, 57-17.

[7] A. Love, S. Middleman and A. K. Hochberg. Journal of Crystal Growth 129, 1993, 119-133.

[8] S. Middleman. Journal of Crystal Growth 114, 1991, 13-21.

[9] C. Vahlas, B. Caussat, P. Serp, G. N. Angelopoulos. Materials Science and Engineering Research 53, 2006, 1-72.

[10] http://webbook.nist.gov/chemistry/ (September 2015).

[11] E. S. Filatov, H. Nizard, P. P. Semyannikov, S. V. Sysoev, S. V. Trubin, N. B. Morozova, K. V. Zherikova, and N. V. Gelfond. Meet. Abstr. 2009 MA2009-02(34): 2532 .

[12] D. A. Labuntsov. High temperatures 5, 1967, 579-647.

[13] R. Marek, J. Straub. International Journal of Heat and Mass Transfer 44, 2001, 39-53.

[14] V. Siriwongrungson, S. Krumdieck. Chemical Vapor Deposition 17, 2011, 1-11. 
[15] R. B. Bird, W. E. Stewart, E. N. Lighfoot. Transport Phenomena (2 ${ }^{\text {nd }}$ Edition). John Wiley and Sons, INC, 2002.

[16] N. R. Gunby, S. Krumdieck, H. Murthy, S. L. Masters, S. S. Miya. Physica Status Solidi a, Vol 212, No. 7, 2015, 1519-1526.

[17] S. Krumdieck and R. Raj. Journal of the American Ceramic Society, 82 [6], 1999, 1605-1607.

[18] C. W. Lim, M. R. Smith, H. M. Cave, M. C. Jermy, J.-S. Wu, S.P. Krumdieck. Computers \& Fluids 86, 2013, 14-27.

[19] D. Lee, S. D. Talwar, S. Krumdieck. Surface and Coating Technology, Vol. 230, 2013 $39-45$.

[20] S. Krumdieck, S. I. Baluti, L. Marcus, and A. Peled. Journal of the Electrochemical Society Proceedings of CVD-XVI/EUROCVD-15 (Bochum, Germany, September 4-9, 2005, 120-127). 